\title{
Task relevance of emotional information affects anxiety-linked attention bias in visual search
}

Article

Accepted Version

Dodd, H. F., Vogt, J., Turkileri, N. and Notebaert, L. (2017) Task relevance of emotional information affects anxiety-linked attention bias in visual search. Biological Psychology, 122. pp. 13-20. ISSN 0301-0511 doi:

https://doi.org/10.1016/j.biopsycho.2016.01.017 Available at https://centaur.reading.ac.uk/55068/

It is advisable to refer to the publisher's version if you intend to cite from the work. See Guidance on citing.

To link to this article DOI: http://dx.doi.org/10.1016/j.biopsycho.2016.01.017

Publisher: Elsevier

All outputs in CentAUR are protected by Intellectual Property Rights law, including copyright law. Copyright and IPR is retained by the creators or other copyright holders. Terms and conditions for use of this material are defined in the End User Agreement.

www.reading.ac.uk/centaur 
Central Archive at the University of Reading

Reading's research outputs online 
RUNNING HEAD: EMOTION RELEVANCE AND ANXIETY-LINKED ATTENTION BIAS

\section{Task relevance of emotional information affects} anxiety-linked attention bias in visual search

Helen F. Dodd +

Julia Vogt+*

Nilgun Turkileri ${ }^{+}$

University of Reading

Lies Notebaert\#

University of Western Australia

* Corresponding author (email: j.vogt@reading.ac.uk)

+ School of Psychology and Clinical Language Sciences, University of Reading, Earley Gate, Whiteknights, Reading, RG6 6AL, UK (emails: h.f.dodd@reading.ac.uk, j.vogt@reading.ac.uk, n.turkileriinseloz@pgr.reading.ac.uk)

\# University of Western Australia, School of Psychology, 35 Stirling Highway, Crawley, WA 6009, Australia (email: lies.notebaert@uwa.edu.au) 


\begin{abstract}
Task relevance affects emotional attention in healthy individuals. Here, we investigate whether the association between anxiety and attention bias is affected by the task relevance of emotion during an attention task. Participants completed two visual search tasks. In the emotion-irrelevant task, participants were asked to indicate whether a discrepant face in a crowd of neutral, middle-aged faces was old or young. Irrelevant to the task, target faces displayed angry, happy, or neutral expressions. In the emotionrelevant task, participants were asked to indicate whether a discrepant face in a crowd of middle-aged neutral faces was happy or angry (target faces also varied in age). Trait anxiety was not associated with attention in the emotion-relevant task. However, in the emotion-irrelevant task, trait anxiety was associated with a bias for angry over happy faces. These findings demonstrate that the task relevance of emotional information affects conclusions about the presence of an anxiety-linked attention bias. Word count: 150
\end{abstract} Keywords: attention bias; anxiety; visual search; emotion; threat; task relevance; goal; top-down 


\section{Task relevance of emotional information affects}

\section{anxiety-linked attention bias in visual search}

Cognitive models of anxiety propose that dysfunctional cognitions lie at the core of anxiety pathology (e.g. A. T. Beck \& Clark, 1997). A number of models focus specifically on anxiety-linked abnormalities in attention (e.g., Mathews \& MacLeod, 1994; Mogg \& Bradley, 1998; Wells \& Matthews, 1994; Williams, MacLeod, \& Mathews, 1988). Whilst there are some important differences between the precise mechanisms hypothesised in these models, all predict that individuals with high levels of anxiety have a chronic bias to preferentially orient attention to stimuli that are threatening. For example, Mogg and Bradley (1998) propose that individuals high in trait anxiety are more likely to appraise a stimulus as threatening than individuals low in trait anxiety and this in turn affects a goal engagement system, which orients attention toward the threat stimulus. From an evolutionary perspective, this mechanism is attributed to the adaptive function of fear (or anxiety), such that threat should be detected quickly in order to activate immediate defensive responses, which in turn will favour threatrelated stimuli over neutral ones (Bradley, 2009; Öhman, 1996).

Extensive research has examined the association between anxiety and attention bias for threat. The vast majority of this work has employed behavioural tasks including the dot probe, Stroop, spatial cueing, visual search and attentional blink task (e.g., BarHaim, Lamy, Pergamin, Bakermans-Kranenburg, \& van IJzendoorn, 2007; Fox, Russo, \& Georgio, 2007; Koster, Leyman, De Raedt \& Crombez, 2006; Notebaert, Crombez, Van Damme, De Houwer, \& Theeuwes, 2011). Neuroscientific techniques including fMRI, EEG, and tDCS have been used to provide insight into anxiety-related differences in 
attention (e.g., Bar-Haim, Lamy \& Glickman, 2005; Bishop, Duncan, \& Lawrence, 2004; Bishop, Jenkins, \& Lawrence, 2007;Clarke, Browning, Hammond, Notebaert \& Macleod, 2014; Etkin, Klemenhagen, Dudman, Rogan, Hen, Kandel et al. 2004; Monk et al., 2006) and there has recently been a surge of work using eye tracking to capture attention over time in anxious populations (Armstrong \& Olatunji, 2012; Chen, Thomas, Clarke, Hickie, \& Guastella, 2015; Dodd et al., 2014).

Across this range of techniques, there has been some inconsistency in findings but on balance the evidence suggests that anxious adults display an attention bias for threat-related stimuli. A meta-analysis of research using the dot probe, Stroop, and spatial cueing task found that the attention bias is reliably found at a moderate effect size across these tasks (Bar-Haim et al., 2007). More recently, a meta-analysis of eye tracking studies reported an anxiety-related attention bias of similar magnitude (Armstrong \& Olatunji, 2012).

In the present paper, we aim to extend this work by investigating how top-down factors such as task goals that determine the relevance of emotional information in an attention task affect anxiety-linked attention bias. A number of theories of attention predict that goals guide visual attention such that individuals will preferentially attend to stimuli that have relevance for their current goal and disregard stimuli that are irrelevant to these goals, even when goal-relevant stimuli have no long-term emotional or motivational value (Corbetta \& Shulman, 2002; Desimone \& Duncan, 1995; Moskowitz, Li, \& Kirk, 2004; Vogt, De Houwer, Moors, Van Damme, \& Crombez, 2010; Vogt, De Houwer, \& Moors, 2011). A series of studies examining basic emotional attention have recently tested this prediction in healthy samples. These studies have indicated that task instructions and goals can profoundly impact participants' 
performance during tasks measuring attention to emotion. Importantly, the findings suggest that attention bias to emotional information may be absent when emotion is task irrelevant (e.g., Hahn \& Gronlund, 2007; Vogt, De Houwer, Crombez, \& Van Damme, 2013; Vromen, Lipp, \& Remington, 2015).

A nice example of this work comes from Stein and colleagues (Stein, Zwickel, Ritter, Kitzmantel, \& Schneider, 2009) who conducted three versions of the attentional blink task. In this task, participants are presented with a stream of visual stimuli in rapid succession. An 'attentional blink' occurs when participants fail to detect a second target (T2) that occurs within quick succession of a first target (T1), typically less than 500 ms (Shapiro, Arnold \& Raymond, 1997). This task can be used to estimate the attentional resources allocated to the T1 stimulus; with longer blinks associated with increased allocation of attention to T1 (Shapiro et al., 1997). In the three versions of the attentional blink task used by Stein and colleagues, the stimuli were identical, with T1 being an emotional face and T2 being a scene. Participants' task at T2 was always to identify whether the scene was an outdoor or indoor scene but their task at T1 was manipulated across the three versions. In the first experiment, participants were asked to categorise the emotion shown on the face. In the second, they were asked to categorise the gender of the face. In the third, they were given no task for T1. The results indicated that fearful faces induced a stronger attentional blink than neutral faces only in the first experiment, when participants had been instructed to respond to the emotion shown on the face. No difference was found in the attentional blink caused by the fearful relative to neutral faces in the second and third experiment, when facial emotion was not task relevant. The authors interpret their findings as indicating that 
the effect of emotional faces on temporal attention is sensitive to participants' attentional set, which depends on the task goal.

In a similar study using visual search to examine spatial attention, Hodsoll, Viding, and Lavie (2011) examined whether emotional faces would capture attention when they were not relevant to the task. Across a series of five experiments, participants were asked to locate a target face, defined as the discrepant gender in the array, and to report the orientation of the face. Thus emotion was not relevant to the task. On one-third of trials one of the faces displayed an emotional expression; half of the time this was the target face and half of the time it was one of the distractor faces. Evidence for difficulty disengaging attention from emotional distractors was found across fearful, angry and happy stimuli; participants were slower to respond to the target face when the distractor faces included an emotional face. In contrast, there was no indication that negative emotional faces engaged attention; when the array displayed a negative target stimulus with neutral distractors, participants were no faster to respond to the target than when the array displayed a neutral target amongst neutral distractors. A facilitation effect, indicating attentional engagement was found when target faces were happy. Taken together, these results indicate that, in healthy individuals, when emotion is not task relevant, negative emotion does not engage attention. This is consistent with the results of Stein et al. (2009), although emotion may affect disengagement effects of attention from distractors. The former findings may appear to contradict the vast literature using visual search tasks, which demonstrates that emotion engages attention and facilitates target detection (e.g., Pinkham, Griffin, Baron, Sasson, \& Gur, 2010; Williams, Moss, Bradshaw, \& Mattingley, 2005). However, these findings are in fact consistent with those of Stein et al. (2009) and Hodsoll et al. 
(2011) because, in classic visual search studies, emotion is relevant to the task; thus an attention bias for emotional stimuli would be expected also from a top-down perspective.

The research outlined above indicates that the task relevance of emotion can have an important impact on whether a bias in attention is observed in healthy individuals or not. What is currently unclear is how this effect of task instructions and task goals interacts with long-term goals or biases in attention such as those observed in anxious individuals. For instance, attention bias to negative information might be more readily erased or overwritten in healthy participants because they appraise negative emotional events as less dangerous or have not acquired biases towards them. Relatedly, some theories of attention bias in anxiety have suggested that chronically activated long-term goals might underlie attention to threat in anxiety. For example, Wells and Matthews (1994; see also Vogt et al., 2013) propose that the goal to search for threat is habitually active in anxious individuals and that it drives an attention bias for threat even when the task goal of the implemented attention task does not turn threat task relevant. If this is the case then it follows that anxious participants will attend to threat even when it is not their explicit task goal. However, when non-anxious individuals are given a goal of searching for emotion, their attention should resemble that of anxious participants such that anxiety-related differences in attention are minimised. This prediction is based on previous research suggesting that non-anxious participants display an attention bias to threatening information that is equivalent to the bias shown by high anxious individuals when threatening information is worth monitoring such as cues that predict the delivery of an electrocutaneous shock (Notebaert et al., 2011). Thus, an anxiety-linked attention bias may be most apparent 
when the emotional content of stimuli is not relevant to the attention task. This prediction is examined in the present research.

In this study we draw upon the research outlined above, regarding the importance of task instructions and goals, to examine how the relevance of emotion to task goal affects conclusions regarding anxiety-linked attention bias. This is a vital area of study because a wide range of tasks are used to measure attention bias, and the task goals given to participants vary across these tasks. In some paradigms participants are instructed (given the goal) to search for emotion (e.g., Öhman, Flykt, \& Esteves, 2001; Rinck, Becker, Kellerman \& Roth, 2003), other tasks do not give this as an explicit instruction but the task is designed in such a way that participants would quickly establish the goal of looking for emotion (e.g., Notebaert, Crombez, Van Damme, De Houwer, \& Theeuwes, 2010; Notebaert, Crombez, Vogt, De Houwer, Van Damme, \& Theeuwes, 2011), and in others, like the dot probe task, emotion is entirely irrelevant to the task (MacLeod, Mathews, \& Tata, 1986). Despite these differences between tasks, to our knowledge, no research has examined how the emotional relevance of task goals affects attention bias associated with anxiety. This research will therefore have implications for the design and interpretation of studies focused on attention bias in anxiety.

We examine the association between anxiety and attention bias to threat using two consecutive visual search tasks, which differ on task goal. In one task, participants are given the goal of searching for an emotional face (emotion-relevant task). In the other task, emotion is present but is not the search goal (emotion-irrelevant task). Based on theories of attention bias that predict high anxious participants have an enduring bias to selectively attend to threat (e.g., Mogg \& Bradley, 1998; Wells \& 
Matthews, 1994; Williams et al., 1988, 1997), we predict that anxiety will be positively associated with a bias for angry relative to happy target faces, when the search goal is emotion irrelevant (emotion-irrelevant task), indicating that, as anxiety levels increase, angry faces engage attention faster than happy faces. On the other hand, we predict that all participants will orient their attention to the emotional faces when emotion is the search goal (emotion-relevant task), and there will, therefore, be no association between anxiety and search speed for angry relative to happy faces in the emotionrelevant task.

\section{Method}

\section{Participants}

Forty-two undergraduate and master's students (40 female, 2 male) with a mean age of 20.40 years ( $S D=3.46$ years) participated. All except two were right-handed. Most of the participants had British nationality $(N=32)$ or were of other European nationality $(N=9)$. Participants were either recruited from an undergraduate participation pool and received course credit in return for participation or were invited to the study via word of mouth.

\section{Apparatus and Stimuli}

\section{Questionnaires.}

Participants completed a basic demographic form (age, gender, nationality, and handedness) and the Spielberger State Trait Anxiety Inventory (STAI; Spielberger, Gorsuch, Lushene, Vagg, \& Jacobs, 1983). The trait form of the STAI (STAI-T) measures relatively stable individual differences in anxiety. Participants are asked to use a 4-point scale (1="Almost never", 2="Somewhat" 3="Often" and 4="Almost always") to indicate how they generally feel (e.g., "I feel secure", "I feel inadequate”, "I feel nervous and 
restless"). The state form of the STAI (STAI-S) measures the intensity of current anxiety (e.g., "I feel calm”, "I feel at ease”, "I am jittery"). Participants are asked to use a 4-point scale (1="Not at all", 2="Somewhat" 3=" Moderately so" and 4="Very much so") to indicate how they feel at that moment. Both forms consist of 20 items with higher scores indicating higher levels of anxiety. Due to a programming error, the items of the STAI-S form were presented in random order. In the present sample, STAI-T scores ranged from 25 to 65 , with a mean score of $43.31(S D=10.24)$. This is slightly higher than the published norms for college students but is within half a standard deviation (Spielberger, Gorsuch, Lushene, Vagg, \& Jacobs, 1983). Internal consistency was good ( $\alpha$ $=.94$ for STAI-T and $\alpha=.93$ for STAI-S).

\section{Visual Search Tasks.}

\section{Lab equipment.}

The visual search task was conducted on an ASUS DualCore computer with a 19" colour monitor (60Hz refresh rate) with a standard QWERTY keyboard. The experiment was programmed and data were collected using E-Prime 2.0 Software (Psychology Software Tools, 2012).

\section{Stimuli.}

All pictures were selected from the FACES database (Ebner, Riediger, \& Lindenberger, 2010), which contains images of young, middle-aged and older adults displaying a range of emotional expressions. Images in this database are standardised with regards the size and alignment of the head, colour and brightness. Validation work by Ebner and colleagues indicates that young adults can accurately differentiate the age of the actors in each age group and identify the happy faces in this database with $97 \%$ accuracy and angry faces with 93\% accuracy. From this database, we selected old, 
young, and middle-aged actors (16 individuals for each age group, 48 individuals in total). For every age group, eight female and eight male actors were selected. For each actor, we chose one angry, one happy, and one neutral emotional expression (144 pictures in total). Using Adobe Photoshop CS2ll, stimuli were converted to gray scale and the hair and ears were cropped using a circular template.

\section{Design}

\section{Visual search tasks}

The basic task parameters were identical for both visual search tasks. A trial began with the presentation of a black fixation cross in the centre of a white screen for $1000 \mathrm{~ms}$. The fixation cross was then replaced by an array of 8 images, arranged in a circle such that each image was equidistant from the centre of the screen (see Figure 1).

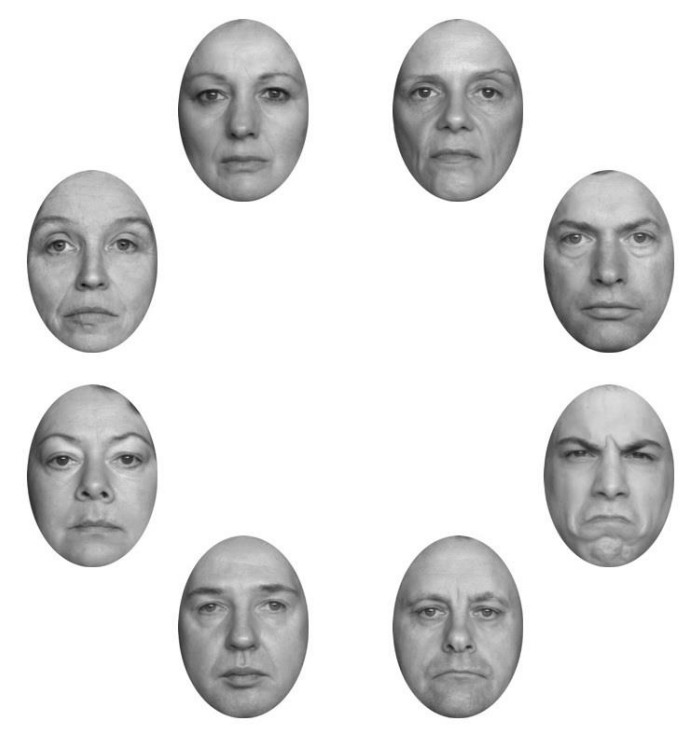




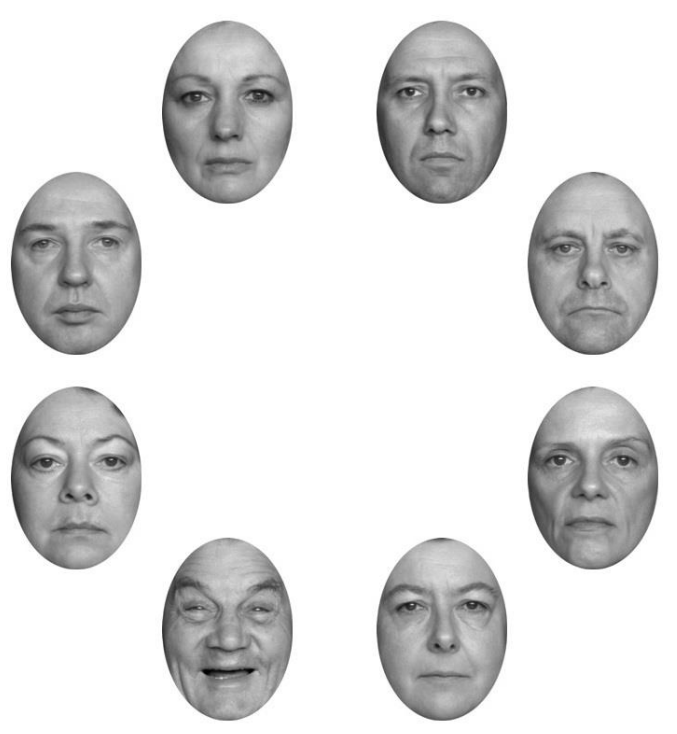

Figure 1. Example stimuli arrays showing an angry-young target in an array of neutral middle-aged faces (top) in the emotion-irrelevant task and a happy-old target in an array of neutral middle-aged faces in the emotion-relevant task (bottom).

The distance from the fixation point at the centre of the display to the centre of each one of the eight faces was $8 \mathrm{~cm}$. The size of each picture was $3.6 \times 5.1 \mathrm{~cm}(136 \mathrm{x}$ 193 pixels or approximately $\left.5.2^{\circ} \times 6.3^{\circ}\right)$. The size of the whole display on the screen was $18.3 \times 19.8 \mathrm{~cm}\left(691 \times 748\right.$ pixels or approximately $\left.25.8^{\circ} \times 24^{\circ}\right)$. The array of faces was displayed for $6000 \mathrm{~ms}$ or until the participant made a response. When participants made an error, the word "Incorrect" was presented for 500ms as feedback; no feedback was given on correct trials. Trials were separated by an inter trial interval of $1000 \mathrm{~ms}$.

The Emotion-Irrelevant Task was configured such that the task relevant dimension was emotion irrelevant. As such, the goal was to identify whether the non- 
middle aged face present was an old or a young face. The target faces varied in emotional expression, but this was irrelevant to the task. On each trial, the array displayed seven distractor images, which were always neutral and middle aged, and one target face.

The target face was either old or young. The task consisted of 192 trials. A target was present on every trial. In half the trials, the target was old and in the other half of trials the target was young. For the majority of trials, the target face displayed a neutral expression (128 trials). However, on a minority of trials, the target face displayed a happy (32 trials) or angry (32 trials) expression. Emotional faces were only present for a minority of trials to ensure that an emotional search strategy was not an efficient way for participants to complete the task. Each array included an equal number of male and female faces. Eight different actors were seen on each trial. Target position was randomized across trials.

In the Emotion-Relevant Task the task relevant dimension was emotion. While the target faces could also vary in age, this was irrelevant to the task. The task goal was to identify whether the non-neutral face present was an angry or a happy face. This task also included 192 trials. A target was present on every trial, and was an angry face on half the trials and a happy face on the other half. The crowd was always middle-aged and neutral. To match the design of the emotion-irrelevant task, for the majority of trials the target was also middle aged (128 trials) and for a minority of trials, the target was old (32 trials) or young (32 trials). Each array included an equal number of male and female faces and eight different actors were seen on each trial. Target position was randomized for each participant.

\section{Procedure}


All participants provided written informed consent prior to the study, which was approved by the ethics committee of the School of Psychology and Clinical Language Sciences, University of Reading.

All tasks, including the questionnaires, were completed on the lab PC. Participants sat approximately $50 \mathrm{~cm}$ in front of the screen and first completed the demographic form and then the STAI-S and STAI-T, respectively. Next, they completed the two visual search tasks: First the emotion-irrelevant task and then the emotionrelevant task. The tasks were not counterbalanced to ensure that there was no carry over effect from the emotional goal in the emotion-relevant task to the emotionirrelevant task.

Participants completed 16 practice trials before each task. In the emotionirrelevant task, participants were informed that seven of the faces were the same age, but one of them was a different age. They were told that their task was to indicate whether the discrepant face was an old or a young individual by pressing "o" for old or "y" for young on the keyboard.

After a short break, participants proceeded to the emotion-relevant task. In this task, participants were informed that seven of the faces were posing the same facial expression, but one of them would be different, and their task was to detect whether the discrepant face was an angry or happy facial expression pressing "a" for angry or " $h$ " for happy on the keyboard. Hereafter, participants were debriefed.

\section{Data preparation}

Data from two participants were not available because of a recording error with the program. For the remaining participants, incorrect trials were excluded from the analysis (9.69\%). In addition, responses that were two standard deviations faster or 
slower than a participants' average response time, calculated for each combination of target emotion and target age for the two tasks separately, were also removed $(4.34 \%)$. Analyses were based on reaction time (RT) data. Greenhouse-Geisser corrected tests of Within-Subjects effects are reported.

\section{Results}

Participants performed significantly more accurately in the emotion-relevant task $(M=94.82 \%, S D=3.82 \%)$ as compared to in the emotion-irrelevant task $(M=$ $85.8 \%, S D=6.91 \%), t(39)=8.47, p<.001$. In addition, average RTs were significantly shorter in the emotion-relevant task $(M=1220 \mathrm{~ms}, S D=320 \mathrm{~ms})$ as compared to the emotion-irrelevant task ( $M=1955 \mathrm{~ms}, S D=406 \mathrm{~ms}), t(39)=14.43, p<.001$. Neither RT nor accuracy on either task were correlated significantly with trait or state anxiety, $p>$ .070 (largest $r=-.29$ ). As our central hypothesis concerns the influence of the task relevance of emotion on the relationship between dispositional anxiety and attention bias, we performed the following analyses using STAI Trait scores. The STAI Trait and State scores were highly correlated, $r(40)=.766, p<.001$.

In the Emotion-Relevant Task, the task-relevant dimension was emotion, as participants were asked to indicate if the non-neutral face displayed a happy or angry expression. The task-irrelevant dimension was age, as the target could be a young, middle-aged, or old face. To examine the contribution of trait anxiety to the allocation of attention to emotional faces when emotion is task relevant, a Target Emotion (Happy, Angry) by Target Age (Young, Middle-aged, Old) Repeated Measures ANOVA was conducted on target identification reaction times with Trait Anxiety scores as a covariate. The only significant effect to emerge was the main effect of Target Emotion, with faster reaction times to happy faces $(M=1014 \mathrm{~ms}, S D=286 \mathrm{~ms})$ than to angry faces 
$(M=1301 \mathrm{~ms}, S D=360 \mathrm{~ms}), F(1,76)=22.20, p<.001, \mathrm{etap}^{2}=.37$. All other effects were non-significant, $p>$.1. Importantly, none of the interactions with Trait Anxiety, nor its main effect, reached significance, $p s>.12$ (largest $F=2.59$ ) indicating no significant impact of trait anxiety on attentional allocation to angry and happy faces when emotion is task relevant. For an overview of the average reaction time to each target, please refer to Table 1.

Table 1. Mean and standard deviations of reaction times (in ms) to target by target type and task.

\section{Emotion-Relevant Task}

\begin{tabular}{|c|c|c|c|c|c|c|}
\hline \multirow[b]{3}{*}{$\begin{array}{l}\text { Relevant } \\
\text { dimension }\end{array}$} & \multicolumn{6}{|c|}{ Irrelevant dimension } \\
\hline & \multicolumn{2}{|c|}{ Young } & \multicolumn{2}{|c|}{ Middle-aged } & \multicolumn{2}{|c|}{ Old } \\
\hline & $M$ & $S D$ & $M$ & $S D$ & $M$ & $S D$ \\
\hline Angry & 1390 & 411 & 1282 & 318 & 1231 & 419 \\
\hline Happy & 1010 & 317 & 1010 & 272 & 1020 & 311 \\
\hline
\end{tabular}

\section{Emotion-Irrelevant Task}

Irrelevant dimension

\begin{tabular}{lcccccc} 
& \multicolumn{2}{c}{ Angry } & \multicolumn{2}{c}{ Neutral } & \multicolumn{2}{c}{ Happy } \\
Relevant & $M$ & $S D$ & $M$ & $S D$ & $M$ & $S D$ \\
dimension & & & & & & \\
\hline Young & 1957 & 482 & 1982 & 443 & 1643 & 379 \\
Old & 1571 & 472 & 1850 & 541 & 1619 & 427
\end{tabular}

In the Emotion-Irrelevant Task, the task relevant dimension was age, as participants were asked to indicate whether the non-middle-aged face depicted an old 
or young person. The task-irrelevant dimension was emotion, as the target could be depicted with a happy, angry, or neutral expression. To test our prediction that trait anxiety will be associated with increased attentional selectivity to angry faces when emotion is task irrelevant, a Target Age (Young, Old) by Target Emotion (Angry, Neutral, Happy) Repeated Measures ANOVA was conducted on target identification reaction times, with Trait Anxiety scores as a covariate.

Results showed significant main effects of Target Emotion, $F(2,37)=8.26, p=$ .001 etap $^{2}=.18$, as well as a significant interaction between Target Age and Target Emotion, $F(2,37)=3.43, p=.045$, eta $^{2}=.08$. This interaction reflected a significant reaction time advantage for both happy faces and angry faces as compared to neutral faces in the old faces, $t(39)=3.53, p=.001$, and $t(39)=5.86, p<.001$, respectively, whereas there was only a reaction time advantage for happy faces in the young faces, and not for angry faces, $t(39)=5.72, p<.001$ and $t(39)=-0.60, p=.551$, respectively. Importantly, there was also a two-way interaction between Target Emotion and Trait Anxiety, $F(2,37)=3.80, p=.030$, etap $^{2}=.09$, which was not further subsumed under the interaction with Target Age, $F(2,37)=1.41, p=.25$, etap $^{2}=.04$. To examine this two-way interaction, we computed an Attention Bias Index (ABI), reflecting speeded responding to angry faces as compared to happy faces, by subtracting reaction times for angry face target trials from reaction times for happy face target trials (across all ages). A positive $\mathrm{ABI}$ score thus indicates an attention bias for angry relative to happy faces. To investigate the nature of the interaction between Target Emotion and Trait Anxiety, these ABI scores were correlated with Trait Anxiety scores. Results showed a significant positive correlation, $r(40)=.33, p=.037$, indicating that, consistent with our prediction, higher levels of trait anxiety were associated with a greater attention bias to angry over 
happy faces. This association can be seen in Figure 2. None of the other main effects or interactions reached significance, $p s>.1$. To examine whether this correlation is driven by speeding to angry faces or slowing to happy faces, two additional ABI's were created, one subtracting reaction times to happy faces from reaction times to neutral faces ( $\left.\mathrm{ABI}_{\text {happy }}\right)$, and one subtracting reaction times to angry faces from reaction times to neutral faces (ABIangry). As trait anxiety showed a significant negative correlation with $\mathrm{ABI}_{\text {happy }}, r(40)=-.350, p=.027$, but not with $\mathrm{ABI}_{\text {angry }}, r(40)=-.052, p=.751$, it appears that the anxiety-linked attention bias is driven by a slowing to happy faces rather than a speeding to angry faces. For an overview of the average reaction times to each target, please refer to Table 1.

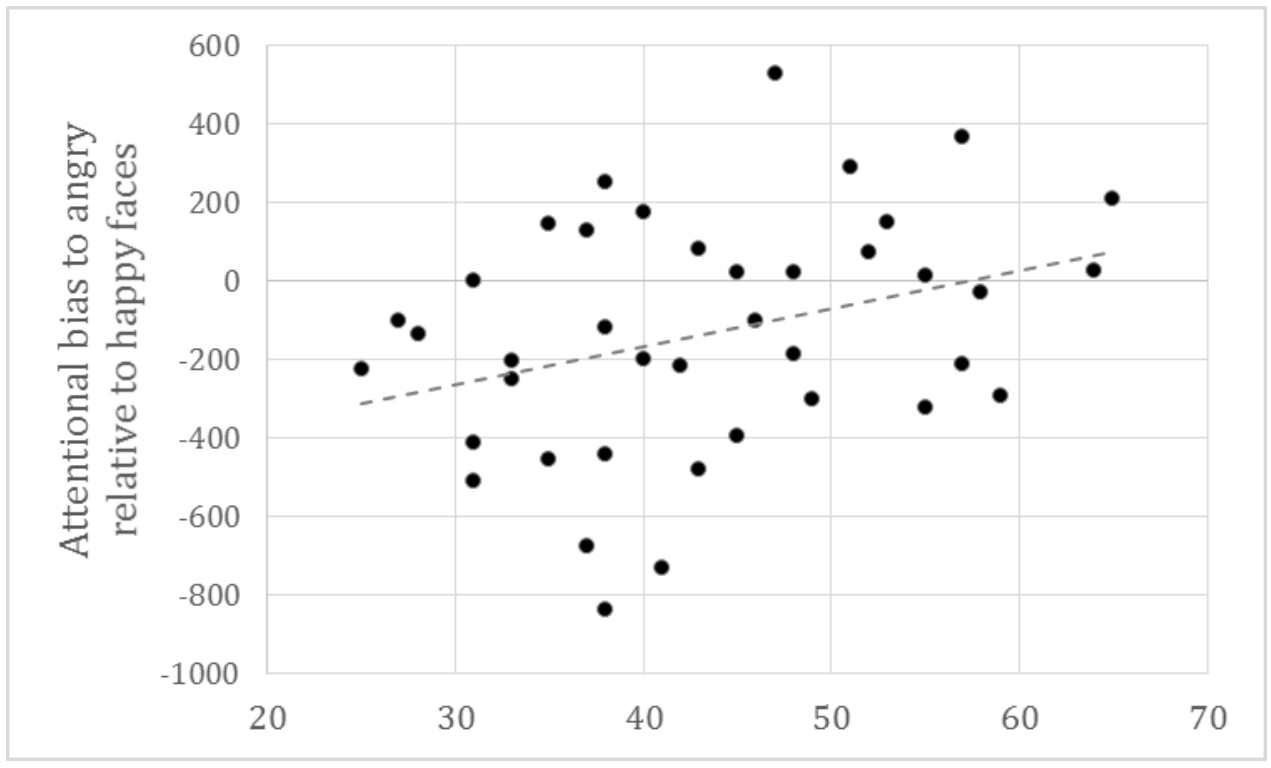

Figure 2. Scatterplot showing the association between trait anxiety (STAI-T scores on xaxis) and attention bias to angry relative to happy faces (in ms, y-axis) on the emotionirrelevant task. 
To directly compare whether the type of task and thus the task relevance of emotion affected attention bias, an $\mathrm{ABI}$ for the emotion-relevant task was first created, again by subtracting reaction times for angry face target trials from reaction times for happy face target trials (across both ages). A paired-sample t-test showed that the ABI was significantly larger in the emotion-irrelevant task $(M=-132.75 \mathrm{~ms}, S D=302.50$ $\mathrm{ms})$, as compared to the emotion-relevant task ( $M=-287.24 \mathrm{~ms}, S D=135.23 \mathrm{~ms}), t(39)$ $=3.506, p=.001$, Cohen's $d=0.640$.

\section{Discussion}

The present study examined the allocation of attention to emotional faces across two visual search tasks that varied with regards to the relevance of emotional facial expression to the task goal. In line with our hypotheses, both high and low anxious participants displayed an attention bias to emotional faces when emotion was task relevant. That is, when they were explicitly instructed to identify the emotion displayed on the target face. In contrast, when participants were instructed to identify the age of the target face, making the emotional value of the face task irrelevant, trait anxiety was associated with enhanced attention to angry relative to happy faces. Notably, the latter effect was driven by slowed responding to happy faces.

These findings are in keeping with recent top-down theoretical accounts of emotional attention (e.g. Corbetta \& Shulman, 2002; Okon-Singer, Lichtenstein-Vidne, \& Cohen, 2013; Pessoa \& Adolphs, 2010; Pourtois, Schettino, \& Vuilleumier, 2013) and attention bias in anxiety (e.g. Wells \& Matthews, 1994). Whereas previous theorizing has predominantly proposed that attention to emotional events is independent of an individual's goals and driven by bottom-up mechanisms, these models suggest a crucial role for goals in driving and controlling attention to emotional events. For instance, 
Pessoa and Adolphs (2010) reviewed evidence suggesting that the (automatic) processing of emotional events depends heavily on higher-order cortical structures, such that interactions between frontal, parietal, and sensory areas control the allocation of attentional resources to task-relevant locations or objects mediating voluntary control (e.g., Corbetta \& Shulman, 2002; Pourtois, Dan, Grandjean, Sander, \& Vuilleumier, 2005; Vuilleumier, Richardson, Armony, Driver, \& Dolan, 2004; Ochsner and Gross, 2005) . Thus, healthy individuals would be expected to attend to emotional stimuli in a similar manner to anxious individuals when current top-down goals promote attention to emotion. From a functional perspective, this would be highly adaptive because it would allow non-anxious individuals to rapidly detect emotional information but only when they are in an environment that requires it (cf. Mogg \& Bradley, 1998). From this perspective, it is not surprising that the attention bias to emotional stimuli in low-anxious participants is reduced when emotion is task irrelevant (cf. Hahn \& Gronlund, 2007; Hodsoll et al., 2011).

In contrast, high-anxious individuals may have chronically activated threat- or emotion-related goals (Wells \& Matthews, 1994) that serve as background goals, leading to an attention bias even when the environment does not require it or when emotion is not task relevant (cf. Vogt et al., 2013). This same pattern of results could also be found if anxious individuals have an enduring bias for threat due to highly sensitive threat detection and evaluation processes, as suggested by most models of attention to threat in anxiety (see Cisler \& Koster, 2010, for an overview); our data do not permit differentiation between these theories. Similarly, recent accounts from affective neuroscience suggest that anxious individuals might be less able to recruit prefrontal resources to task-relevant information (Bishop et al., 2007; Bishop et al., 
2004; Etkin, Egner, \& Kalisch, 2011). This could explain why higher levels of trait anxiety were associated with a bias towards angry faces, when there was clearly a different goal such as in the emotion-irrelevant task. However, it is not possible to ascertain the extent to which performance differences between individuals across the two tasks were driven by amygdala-based bottom-up pathways or frontal top-down pathways (e.g. D. M. Beck \& Kastner, 2009; but see Pourtois et al., 2013; Calder, Ewbank, \& Passamonti, 2011) without combining this type of research with neuroimaging techniques. What our data show is that task goals have an important influence on attention such that low anxious individuals preferentially attend to emotion at a level comparable to high anxious individuals when given an emotional search goal but high anxious individuals preferentially attend to emotion even when emotion is not task relevant.

Although our findings are in keeping with theories of goal-driven attention, as outlined above, it is important to consider an alternative explanation for our findings. Specifically, recent research has suggested that the detrimental effects of anxiety become most apparent under cognitively demanding situations (Eysenck, Derakshan, Santos, \& Calvo, 2007; Berggren \& Derakshan, 2013). In the present study, reaction times suggest that task demand was likely higher in the emotion-irrelevant task. From this perspective, one could argue that no association between anxiety and attention bias was found on the emotion-relevant task because only the emotion-irrelevant task was sufficiently taxing to reveal such effects. There are a number of difficulties with this explanation however. First, reaction times suggest the emotion-relevant task was not particularly easy, and one could argue that it was significantly more cognitively demanding than many other tasks used to assess attention bias such as the dot probe 
task. Second, the emotion-relevant task was always completed second so one would expect that participants would find it relatively demanding cognitively. Third, in both tasks, overall performance was not affected by trait anxiety. Specifically, higher trait anxiety was associated with fewer errors on the emotion-relevant task and faster responses on the emotion-irrelevant task (although non-significant). Finally, the findings related to cognitive load and attention bias are not consistent, with some studies suggesting that attention to threat only becomes apparent when task demand is low (Pessoa, McKenna, Gutierrez, \& Ungerleider, 2002).

The present results have important implications for research investigating attention to emotional stimuli and in particular for future studies focusing on individual differences in anxiety vulnerability and attention bias for threat. Specifically, our results suggest that an anxiety-linked attention bias will be most apparent when the emotional value of stimuli is not relevant to the task at hand and, indeed, may not be observed at all for tasks that have a salient emotion-relevant goal. This is an important consideration because, as described previously, a range of tasks are used to assess attention bias and these tasks differ with respect to the task relevance of emotion. Furthermore, emotional stimuli can be presented as task relevant or irrelevant within most paradigms that are used to measure attention bias towards emotional stimuli. For instance, similar to the manipulation we used in the present study (also cf. Theeuwes, 1991; Notebaert et al., 2010), emotional stimuli can be presented as distractors but also as targets in the attentional blink paradigm (Stein et al., 2010) or response interference tasks (Van Dillen, Lakens, \& Van den Bos, 2011). Thus inconsistencies in findings may occur as a result of differences in the task relevance of emotion. 
Relatedly, recent research has shown that goals can affect attention, even when the goal is induced for a parallel task; when participants are asked to switch between two independent tasks, goals induced by the task parameters of one task can affect the allocation of attention on a second task. Thus, if emotion is relevant for task A (a nonattentional task), this can lead to an attention bias for emotion in task B (an attention task), even if emotion is not relevant to the goal of task B (e.g., Notebaert, Crombez, Vogt et al., 2011; Vogt et al., 2010; Vogt et al., 2013; also cf. Bacon and Egeth, 1994; Folk, Remington, \& Johnston, 1992). Hence, for top-down influences to occur, participants do not necessarily need to be completing a task that implements an intention to attend to events with emotional value (i.e. to search for emotional faces) but it is sufficient to have a 'background' goal (i.e. identify emotional events in a parallel or preceding nonattentional task) to which emotional events are relevant. In a related vein, emotional events can become relevant through other processes such as when information is presented in a task-relevant location (Lichtenstein-Vidne, Henik, \& Safadi, 2012). Hence, emotional information can become (task) relevant due to various aspects of the task design and not only by instructing participants explicitly to attend to emotional information (see also Kanske, 2012). It is therefore important to carefully consider such carry-over and indirect effects in the design of a task or study.

\section{Limitations}

The present study is not without its limitations and these provide grounds for further research in this area. First of all, we hope that future research will attempt to replicate the present findings, ideally also testing clinically anxious individuals (cf. Mogg, Philippot \& Bradley, 2004). In a related vein, future research should also continue to investigate the effects of anxiety on the processing of positive emotional information. 
Further, in the present study, we attempted to match the two versions of the task as much as possible. However, as already discussed, the emotion-irrelevant task was more difficult than the emotion-relevant task most likely because classifying age was challenging. We used age as the emotional-irrelevant dimension because it represents a face-inherent characteristic. If we would have superimposed stimuli on the faces such as an artificial hair line for the discrimination task we would have used an artificial stimulus that is very different from the emotional facial expressions which represent rather naturalistic features of faces. However, future research might aim to attempt other features such as race (Lipp, Craig, Frost, Terry, \& Smith, 2014) that are easier to identify. Importantly, age (but perhaps even more so race) is also not entirely emotional irrelevant with old age being perceived as negative and young age as positive (Ebner, 2008). This might for instance explain why there was a search advantage for old angry but not young angry faces in the emotion-irrelevant task as old, angry faces combine two negative features which might facilitate search (cf. Hugenberg, 2005). In this context, it is also interesting to note that age only seems to impact attention allocation when it is task relevant as would be predicted by top-down accounts of attention (Folk et al., 1992). Additionally, faster responding to young, happy faces in this task could also reflect an own-age bias (cf. Wiese, Komes, \& Schweinberger, 2013) since our participants were younger adults. Second, our results revealed an attention bias to happy faces in the emotional version of the task. This is in line with many studies suggesting an advantage for happy over negative facial expressions (e.g., Becker, Anderson, Mortensen, Neufeld, \& Neel, 2011; Hodsoll et al., 2011). One reason for this is that happy emotional images are more likely to display teeth, which may attract attention. One could argue that we did not find an effect of trait anxiety in the emotion- 
relevant task because participants translated the task goal into an emotion-irrelevant goal (i.e. search for teeth) and did not process the emotional content of the faces. However, this seems an unlikely explanation for our findings overall because we found an effect of trait anxiety in the emotion-irrelevant task, which explicitly instructed participants to focus on an emotion-irrelevant feature. Finally, symptoms of depression were not assessed in the present study. Given the high comorbidity between anxiety and depression (e.g., Mineka, Watson, \& Clark, 1998), it remains possible that the findings are driven by comorbid symptoms of depression. We feel this is unlikely given that the evidence that individuals with depression also exhibit an attention bias for emotional faces is not as convincing as that for anxiety (Mogg \& Bradley, 2005). There are also methodological and theoretical issues with attempting to tease apart the effects of anxiety and depression (Miller \& Chapman, 2001).

\section{Conclusion}

The present findings showcase how manipulating the task relevance of emotional information can affect conclusions regarding anxiety-linked attention bias for threat. We hope that future research will build on this work by further investigating the underlying mechanisms of attention to emotion as well as the contexts within which attention bias is most salient in healthy and anxious individuals. Ultimately, this line of work may inform the development of procedures that allow people to control (maladaptive) attention to threat. 


\section{References}

Armstrong, T., \& Olatunji, B. O. (2012). Eye tracking of attention in the affective disorders: a meta-analytic review and synthesis. Clinical Psychology Review, 32, 704-723.

Bacon, W. F., \& Egeth, H. (1994). Overriding stimulus-driven attentional capture. Perception and Psychophysics, 55, 485-496.

Bar-Haim, Y., Lamy, D., \& Glickman, S. (2005). Attention bias in anxiety: A behavioral and ERP study. Brain and Cognition, 59, 11-22.

Bar-Haim, Y., Lamy, D., Pergamin, L., Bakermans-Kranenburg, M. J., \& van IJzendoorn, M. H. (2007). Threat-related attention bias in anxious and non anxious individuals: A meta-analytic study. Psychological Bulletin, 133, 1-24.

Beck, A. T., \& Clark, D. A. (1997). An information processing model of anxiety: Automatic and strategic processes. Behaviour Research and Therapy, 35, 49-58.

Beck, D. M., \& Kastner, S. (2009). Top-down and bottom-up mechanisms in biasing competition in the human brain. Vision Research, 49, 1154-1165.

Becker, D. V., Anderson, U. S., Mortensen, C. R., Neufeld, S. L., \& Neel, R. (2011). The face in the crowd effect unconfounded: Happy faces, not angry faces, are more efficiently detected in single-and multiple-target visual search tasks. Journal of Experimental Psychology: General, 140, 637.

Berggren, N., \& Derakshan, N. (2013). Attentional control deficits in anxiety: Why you see them and why you don't. Biological Psychology, 92, 440-446. 
Bishop, S. J., Duncan, J., Lawrence, A. D., 2004. State anxiety modulation of the amygdala response to unattended threat-related stimuli. Journal of Neuroscience, 24, 10364-10368.

Bishop, S. J., Jenkins, R., Lawrence, A. D., 2007. Neural processing of fearful faces: effects of anxiety are gated by perceptual capacity limitations. Cerebral Cortex, 17, 1595-1603.

Bradley, M., 2009. Natural selective attention: orienting and emotion. Psychophysiology $46,1-11$.

Calder, A. J., Ewbank, M., \& Passamonti, L. (2011). Personality influences the neural responses to viewing facial expressions of emotion. Philosophical Transactions of the Royal Society B: Biological Sciences, 366, 1684-1701.

Chen, N. T. M., Thomas, L. M., Clarke, P. J. F., Hickie, I. B., \& Guastella, A. J. (2015). Hyperscanning and avoidance in social anxiety disorder: The visual scan path during public speaking. Psychiatry Research, 225, 667-672.

Cisler, J. M. \& Koster, E. H. W. (2010). Mechanisms of attention biases towards threat in anxiety disorders: An integrative review. Clinical Psychology Review, 30, 203-216.

Clarke, P. J., Browning, M., Hammond, G., Notebaert, L., \& MacLeod, C. (2014). The causal role of the dorsolateral prefrontal cortex in the modification of attention bias: Evidence from transcranial direct current stimulation. Biological Psychiatry, 76, 946-952.

Corbetta, M., \& Shulman, G. L. (2002). Control of goal-directed and stimulus-driven attention in the brain. Nature Reviews Neuroscience, 3, 201-215.

Desimone, R., \& Duncan, J. (1995). Neural mechanisms of selective visual attention. Annual Review of Neuroscience, 18, 193-222. 
Dodd, H. F., Hudson, J. L., Williams, T., Morris, T., Lazarus, R. S., \& Byrow, Y. (2014). Anxiety and attention bias in preschool-aged children: An eyetracking study. Journal of Abnormal Child Psychology, 39, 939-951.

Ebner, N. C. (2008). Age of face matters: Age-group differences in ratings of young and old faces. Behavior Research Methods, 40, 130-136.

Ebner, C., Riediger, M., \& Lindenberger, U. (2010). FACES--a database of facial expressions in young, middle-aged, and older women and men: development and validation. Behavior Research Methods, 42, 351-362.

Etkin, A., Egner, T., \& Kalisch, R. (2011). Emotional processing in anterior cingulate and medial prefrontal cortex. Trends in Cognitive Sciences, 15, 85-93.

Etkin, A., Klemenhagen, K. C., Dudman, J. T., Rogan, M. T., Hen, R., Kandel, E. R., Hirsch, J., 2004. Individual differences in trait anxiety predict the response of the basolateral amygdala to unconsciously processed fearful faces. Neuron, 44 , 1043-1055.

Eysenck, M. W., Derakshan, N., Santos, R., \& Calvo, M. G. (2007). Anxiety and cognitive performance: attentional control theory. Emotion, 7, 336-353.

Folk, C. L., Remington, R. W., \& Johnston, J. C. (1992). Involuntary covert orienting is contingent on attentional control settings. Journal of Experimental Psychology: Human Perception and Performance, 18, 1030-1044.

Fox, E., Russo, R., \& Georgiou, G. A. (2005). Anxiety modulates the degree of attentive resources required to process emotional faces. Cognitive, Affective, \& Behavioral Neuroscience, 5, 396-404.

Hahn, S., \& Gronlund, S. D. (2007). Top-down guidance in visual search for facial expressions. Psychonomic Bulletin \& Review, 14, 159-165. 
Hodsoll, S., Viding, E., \& Lavie, N. (2011). Attentional capture by irrelevant emotional distractor faces. Emotion, 11, 346-353.

Hugenberg, K. (2005). Social categorization and the perception of facial affect: Target race moderates the response latency advantage for happy faces. Emotion, 5, 267276.

Kanske, P. (2012). On the influence of emotion on conflict processing. Frontiers in Integrative Neuroscience, 6, 42.

Koster, E. H. W., Crombez, G., Van Damme, S., Verschuere, B., \& De Houwer, J. (2004). Does imminent threat capture and hold attention? Emotion, 4, 312-317.

Koster, E. H. W., Leyman, L., De Raedt, R., \& Crombez, G. (2006). Cueing of visual attention by emotional facial expressions: The influence of individual differences in anxiety and depression. Personality and Individual Differences, 41, 329-339. Lichtenstein-Vidne, L., Henik, A., \& Safadi, Z. (2012). Task relevance modulates processing of distracting emotional stimuli. Cognition and Emotion, 26, 42-52.

Lipp, O. V., Craig, B. M., Frost, M. J., Terry, D. J., \& Smith, J. R. (2014). Searching for emotion or race: Task-irrelevant facial cues have asymmetrical effects. Cognition and Emotion, 28, 1100-1109.

MacLeod, C., Mathews, A., \& Tata, P. (1986). Attention bias in emotional disorders. Journal of Abnormal Psychology, 95, 15-20.

Mathews, A., \& MacLeod, C. (1994). Cognitive approaches to emotion and emotional disorders. Annual Review of Psychology, 45, 25-50.

Mathews, A., \& Mackintosh, B. (1998). A cognitive model of selective processing in anxiety. Cognitive Therapy and Research, 22, 539-560. 
Mogg, K., \& Bradley, B. P. (1998). A cognitive-motivational analysis of anxiety. Behaviour Research and Therapy, 36, 809-848.

Mogg, K., Philippot, P., \& Bradley, B. P. (2004). Selective attention to angry faces in clinical social phobia. Journal of Abnormal Psychology, 113, 160-165.

Monk, C. S., Nelson, E. E., McClure, E. B., Mogg, K., Bradley, B. P., Leibenluft, E., ... \& Pine, D. S. (2006). Ventrolateral prefrontal cortex activation and attention bias in response to angry faces in adolescents with generalized anxiety disorder. American Journal of Psychiatry, 163, 1091-1097.

Moskowitz, G. B., Li, P., \& Kirk, E. R. (2004). The implicit volition model: On the preconscious regulation of temporarily adopted goals. In M. P. Zanna (Ed.), Advances in Experimental Social Psychology (pp. 317-414). San Diego, CA: Academic Press.

Notebaert, L., Crombez, G., Van Damme, S., De Houwer, J., \& Theeuwes, J. (2010). Looking out for danger: An attention bias towards spatially predictable threatening stimuli. Behaviour Research and Therapy, 48, 1150-1154. doi: 10.1016/j.brat.2010.07.013

Notebaert, L., Crombez, G., Van Damme, S., De Houwer, J., \& Theeuwes, J. (2011). Signals of threat do not capture, but prioritize, attention: A conditioning approach. Emotion, 11, 81-89.

Notebaert, L., Crombez, G., Vogt, J., De Houwer, J., Van Damme, S., \& Theeuwes, J. (2011). Attempts to control pain prioritize attention towards signals of pain: An experimental study. Pain, 152, 1068-1073. doi: 10.1016/j.pain.2011.01.020 Ochsner, K. N., \& Gross, J. J. (2005). The cognitive control of emotion. Trends in Cognitive Sciences, 9, 242-249. 
Öhman, A. (1996). Preferential preattentive processing of threat in anxiety:

Preparedness and attentional biases. In R.M. Rapee (Ed.), Current Controversies in the Anxiety Disorders (pp. 253-290). New York: Guilford.

Öhman, A. (2007). Has evolution primed humans to "beware the beast"? Proceedings of the National Academy of Sciences of the United Stated of America, 104, 1639616397.

Öhman, A., Flykt, A., \& Esteves, F. (2001). Emotion drives attention: Detecting the snake in the grass. Journal of Experimental Psychology: General, 130, 466-478.

Öhman, A., \& Mineka, S. (2001). Fears, phobias, and preparedness: Toward an evolved module of fear and fear learning. Psychological Review, 108, 483-522.

Okon-Singer, H., Lichtenstein-Vidne, L. \& Cohen, N. (2013). Dynamic Modulation of Emotional Processing. Biological Psychology, 92, 480-491.

Pessoa, L., \& Adolphs, R. (2010). Emotion processing and the amygdala: From a 'low road' to 'many roads' of evaluating biological significance. Nature Reviews Neuroscience, $11,773-83$.

Pessoa, L., McKenna, M., Gutierrez, E., \& Ungerleider, L. G. (2002). Neural processing of emotional faces requires attention. Proceedings of the National Academy of Sciences of the United Stated of America, 99, 11458-11463.

Pinkham, A. E., Griffin, M., Baron, R., Sasson, N. J., \& Gur, R. C. (2010). The face in the crowd effect: Anger superiority when using real faces and multiple identities. Emotion, 10, 141-146.

Pourtois, G., Dan, E. S., Grandjean, D., Sander, D., \& Vuilleumier, P. (2005). Enhanced extrastriate visual response to bandpass spatial frequency filtered fearful faces: 
Time course and topographic evoked-potentials mapping. Human Brain Mapping, $26,65-79$.

Pourtois, G., Schettino, A., \& Vuilleumier, P. (2013). Brain mechanisms for emotional influences on perception and attention: what is magic and what is not. Biological Psychology, 92, 492-512.

Psychology Software Tools, Inc. [E-Prime 2.0]. (2012). Retrieved from http://www.pstnet.com.

Rinck, M., Becker, E. S., Kellermann, J., \& Roth, W. T. (2003). Selective attention in anxiety: Distraction and enhancement in visual search. Depression and Anxiety, $18,18-28$.

Shapiro, K. L., Raymond, J. E., \& Arnell, K. M. (1997). The attentional blink. Trends in Cognitive Sciences, 1, 291-296.

Spielberger, C. D., Gorsuch, R. L., Lushene, R., Vagg, P. R., \& Jacobs, G. A. (1983). Manual for the State-Trait Anxiety Inventory. Palo Alto, CA: Consulting Psychologists Press.

Stein, T., Zwickel, J., Ritter, J., Kitzmantel, M., \& Schneider, W. X. (2009). The effect of fearful faces on the attentional blink is task dependent. Psychonomic Bulletin \& Review, 16, 104-109.

Theeuwes, J. (1991). Exogenous and endogenous control of attention: The effect of visual onsets and offsets. Perception \& Psychophysics, 49, 83-90.

Van Dillen, L. F., Lakens, D., \& Van den Bos, K. (2011). At face value: Categorization goals modulate vigilance for angry faces. Journal of Experimental Social Psychology, 47, 235-240. 
Vogt, J., De Houwer, J., Crombez, G., \& Van Damme, S. (2013). Competing for attentional priority: temporary goals versus threats. Emotion, 13, 587-598.

Vogt, J., De Houwer, J., Moors, A., Van Damme, S., \& Crombez, G. (2010). The automatic orienting of attention to goal-relevant stimuli. Acta Psychologica, 134, 61-69.

Vogt, J., De Houwer, J., \& Moors, A. (2011). Unintended allocation of spatial attention to goal-relevant but not to goal-related events. Social Psychology, 42, 48-55.

Vromen, J. M. G., Lipp, O. V., \& Remington, R. W. (2015). The spider does not always win the fight for attention: Disengagement from threat is modulated by goal set. Cognition and Emotion, 29, 1185-1196.

Vuilleumier, P., \& Huang, Y.-M. (2009). Emotional attention: Uncovering the mechanisms of affective biases in perception. Current Directions in Psychological Science, 18, 148-152.

Vuilleumier, P., Richardson, M. P., Armony, J. L., Driver, J., \& Dolan, R. J. (2004). Distant influences of amygdala lesion on visual cortical activation during emotional face processing. Nature Neuroscience, 7, 1271-1278.

Wiese, H., Komes, .J \& Schweinberger, S. R. (2013). Ageing faces in ageing minds: A review on the own-age bias in face recognition. Visual Cognition, 21, 1337-1363. Williams, J. M. G., Watts, F. N., MacLeod, C., \& Mathews, A. (1988). Cognitive psychology and emotional disorders. Chichester, UK: Wiley.

Williams, M., Moss, S., Bradshaw, J., \& Mattingley, J. (2005). Look at me, I'm smiling: Visual search for threatening and nonthreatening facial expressions. Visual Cognition, 12, 29-50.

Wells, A. \& Matthews, G. (1994). Attentions and emotions: A clinical perspective. Hove, UK: Erlbaum. 
Yiend, J. (2010). The effects of emotion on attention: A review of attentional processing of emotional information. Cognition and Emotion, 24, 3-47.

Yiend, J., \& Mathews, A. (2001). Anxiety and attention to threatening pictures. The Quarterly Journal of Experimental Psychology A, 54, 665-681. 